\title{
DcSto: carrot Stowaway-like elements are abundant, diverse, and polymorphic
}

\author{
Alicja Macko-Podgorni • Anna Nowicka • \\ Ewa Grzebelus • Philipp W. Simon • \\ Dariusz Grzebelus
}

Received: 5 March 2013/ Accepted: 10 June 2013/Published online: 18 June 2013

(c) The Author(s) 2013. This article is published with open access at Springerlink.com

\begin{abstract}
We investigated nine families of Stowaway-like miniature inverted-repeat transposable elements (MITEs) in the carrot genome, named DcSto 1 to DcSto9. All of them were AT-rich and shared a highly conserved 6 bp-long TIR typical for Stowaways. The copy number of DcSto 1 elements was estimated as ca. 5,000 per diploid genome. We observed preference for clustered insertions of DcSto and other MITEs. Distribution of DcSto 1 hybridization signals revealed presence of DcSto1 clusters within euchromatic regions along all chromosomes. An arrangement of eight regions encompassing DcSto insertion sites, studied in detail, was highly variable among plants representing different populations of Daucus carota. All of these insertions were polymorphic which most likely suggests a very recent mobilization of those elements. Insertions of DcSto near carrot genes and presence of putative promoters, regulatory motifs, and polyA signals within their sequences might suggest a possible involvement of DcSto in the regulation of gene expression.
\end{abstract}

Electronic supplementary material The online version of this article (doi:10.1007/s10709-013-9725-6) contains supplementary material, which is available to authorized users.

A. Macko-Podgorni · A. Nowicka · E. Grzebelus .

D. Grzebelus ( $\square)$

Department of Genetics, Plant Breeding and Seed Science,

University of Agriculture in Krakow, Al. 29 Listopada 54,

31-425 Kraków, Poland

e-mail: dgrzebel@ogr.ar.krakow.pl

P. W. Simon

USDA-ARS Vegetable Crops Research Unit, Department

of Horticulture, University of Wisconsin-Madison,

1575 Linden Drive, Madison, WI 53706, USA
Keywords Transposable elements - MITE - Stowaway . Carrot · Polymorphism

\section{Introduction}

Transposable elements (TEs), DNA segments capable of changing their chromosomal position, are present in genomes of almost all living organisms. In plants, TEs can constitute from ca. $10 \%$ of Arabidopsis thaliana genome (Arabidopsis Genome Initiative 2000) to $85 \%$ of the B73 maize genome (Schnable et al. 2009). Based on the mechanism of transposition, TEs are divided into two groupsclass I: retrotransposons, and class II: DNA transposons. Retrotransposons transpose via an RNA intermediate (a 'copy-and-paste' mechanism) and each transposition event leads to increase of their copy number. DNA transposons (class II) comprise two subclasses, divided with respect to the number of strands that are cleaved during transposition. Class II TEs belonging to subclass 1 are mobilized via a 'cutand-paste' mechanism, where both DNA strands are cleaved at each end during transposition, while mobilization of subclass 2 elements does not require double-strand cleavage (Finnegan 1989; Wicker et al. 2007). Subclass 1 is further divided into two orders, i.e. TIR, characterized by the presence of terminal inverted repeats (TIRs), and Crypton, identified in fungi, devoid of TIR sequences.

Miniature inverted-repeat transposable elements (MITEs), are usually the most abundant group of DNA transposons. They are characterized by a small size ( $>600 \mathrm{bp}$ ) and similarity of their TIRs to the termini of related Class II TIR transposons (Wicker et al. 2007). Stowaway MITEs were described in maize (Bureau and Wessler 1994) as short $(<500$ base pairs), AT-rich, having a potential to form secondary structures and forming 2-nt 'TA' target site duplications 
(TSD) upon insertion. Some Stowaways may provide polyadenylation sites and $c i s$-acting regulatory regions to adjacent genes. Stowaways were identified in both monocots and dicots (Feschotte et al. 2003). Due to similarity of TIRs (5'-CTG CCT CCR T- $3^{\prime}$, where R stands for A or G) and TSD, it was suggested that Stowaway elements could be mobilized in trans by transposases encoded by Tcl/mariner-like DNA transposons (Feschotte and Wessler 2002; Feschotte et al. 2003, 2005; Macas et al. 2005). Also, it was shown that a single source of transposase can interact with Stowaways belonging to several distinct families. The transposase has a relatively weak binding specificity and the function of TIRs might by suppressed or enhanced by an internal sequence of the element (Feschotte et al. 2005; Yang et al. 2009). Excision of mariners and Stowaways leaves a characteristic footprint, which is element-specific, corresponding to the $5^{\prime}$ and $3^{\prime}$ TIR nucleotides and can be of variable length (Lampe et al. 1996). Thus, a previously occupied locus can be distinguished from the ancestral empty site. Stowaway elements are relatively hypomethylated and frequently occur in genic regions (Mao et al. 2000; Takata et al. 2007). Recently, an active Stowaway element was identified in potato. Mobilization took place in the course of leaf protoplast cultures and caused somaclonal variation of skin color of potato tubers (Momose et al. 2010). Availability of genomic sequences significantly increased the number of TE families identified and facilitated their classification. Nevertheless, in species for which sequence data are restricted, new transposons are often identified following the analysis of knock-out mutations caused by TE insertions into coding regions.

Carrot is one of the most important vegetable crops and a major source of carotenoids that are precursors of vitamin A. However, sequencing data for carrot are not extensive and little is known about the organization of the repetitive portion of the carrot genome, including transposable elements. To date, only two families of TEs, i.e. Tdc1 of CACTA superfamily (Ozeki et al. 1997; Itoh et al. 2003) and DcMaster of PIF/Harbinger superfamily (Grzebelus et al. 2007), the latter associated with a family of Tourist-like MITEs named Krak (Grzebelus and Simon 2009), were identified in the carrot genome. In the present study, we investigated carrot Stowaway-like MITEs, DcSto (Daucus carota Stowaway-like), estimated their copy number, identified local rearrangements created upon insertion that pointed at putative functional implications of their presence on adjacent coding regions.

\section{Materials and methods}

Plant materials

DNA was extracted from plants representing carrot diversity, comprising cultivated carrots of different origin and breeding history (supplementary Table 1) previously used for carrot genetic diversity study (Baranski et al. 2011). Full-length DcSto1 elements were amplified from the genomic DNA of three unrelated cultivated carrots $(8503 \mathrm{~B}$, 493B, 70349-F2), three wild carrots (Daucus carota subsp. carota, D. carota subsp. commutatus, D. carota subsp. gummifer) and other closely related Daucus species (D. capillifolius, D. sahariensis).

DNA isolation, cloning and sequencing

Total genomic DNA was isolated from fresh, young leaves using commercial DNeasy Plant Mini Kit (Qiagen) following a manufacturer's protocol. PCR products separated on agarose gels were cut out, purified with Wizard SV Gel and PCR Clean-Up (Promega), cloned into pGEM-T (Promega) and transformed into Escherichia coli, strain DH10B or PEAK ${ }^{\mathrm{TM}}$ Efficiency Competent Cells (GeneMate). From the transformed bacterial cells, identified by blue-white selection, plasmids were extracted using Wizard SV Miniprep kit (Promega). Sequencing reactions were set up with universal primers Sp6 and T7 or PCR-specific primers using Big Dye terminator chemistry (Applied Biosystems) or CEQ ${ }^{\mathrm{TM}}$ DTCS Quick Start Kit (Beckman Coulter) as recommended by manufacturer. Sequencing was carried out on ABI 3700 capillary sequencer (Applied Biosystems) or CEQ8000 capillary DNA sequencer (Beckman Coulter).

Amplification of DcSto1 from the Genomic DNA of Carrot

A primer complementary to the TIR sequence of a DcSto 1 (DcS-TIR) was designed manually based on sequence of DcSto 1 element identified in the Rs locus. To design the DcS-TIR primer, both Stowaway characteristic features, 16 bp consensus TIR sequence and the characteristic TSD, were considered. To amplify full-length DcSto1 elements the reaction was prepared in $10 \mu \mathrm{l}$ and contained $20 \mathrm{ng}$ genomic DNA, $1 \mathrm{mM}$ DcS-TIR primer (5' TAC TCC CTC CGT CCC ACC $3^{\prime}$ ), $0.25 \mathrm{mM}$ dNTPs (Fermentas), $0.5 \mathrm{U}$ Taq DNA polymerase (Fermentas) and $1 \times$ Taq buffer. The amplification profile was as follows: $94{ }^{\circ} \mathrm{C}$ (1 min), 30 cycles of $94{ }^{\circ} \mathrm{C}(30 \mathrm{~s}), 50{ }^{\circ} \mathrm{C}(40 \mathrm{~s}), 68^{\circ} \mathrm{C}(1 \mathrm{~min})$ and $68{ }^{\circ} \mathrm{C}(4 \mathrm{~min})$. PCR products were separated in $1 \%$ agarose gels, purified, cloned, and sequenced.

To identify DcSto insertion in the chxbl gene, intron primers were designed based on the available mRNA sequence (GenBank: DQ192193). A PCR reaction was set up in $10 \mu \mathrm{l}$ containing $10 \mathrm{ng}$ genomic DNA, $1 \mathrm{mM}$ CHXB1-5'F: 5'CCG AAA TGA TAG CTC GGG TA3'

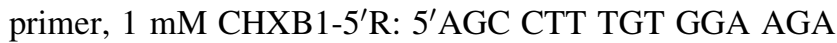
AAC CA3'primer, $0.25 \mathrm{mM}$ dNTPs (Fermentas), $1 \mathrm{U}$ Taq 
DNA polymerase (Fermentas) and $1 \times$ Taq buffer. The amplification profile was as follows: $94{ }^{\circ} \mathrm{C}$ (1 min), 30 cycles of $94{ }^{\circ} \mathrm{C}(30 \mathrm{~s}), 56{ }^{\circ} \mathrm{C}(45 \mathrm{~s}), 68^{\circ} \mathrm{C}(3 \mathrm{~min})$ and a final extension step of $68^{\circ} \mathrm{C}(8 \mathrm{~min})$. Amplified products were separated in $1 \%$ agarose gels, purified, cloned, and sequenced.

\section{Estimation of the Copy Number of DcSto 1}

Copy number of DcSto 1 elements was estimated essentially as proposed by Grzebelus et al. (2006). Two rounds of amplification with DcS-TIR primer were carried out. First PCR was set up to check if at least one element is present per 384-well BAC plate and to confirm the specificity of amplification. For this purpose, pools by plate of B8503 carrot genomic BACs (Cavagnaro et al. 2009) were used. PCR reaction contained 10-30 ng BAC DNA, $1 \mathrm{mM}$ DcS-TIR primer (5' TAC TCC CTC CGT CCC ACC $3^{\prime}$ ), $0.25 \mathrm{mM}$ dNTPs (Fermentas), $0.5 \mathrm{U}$ Dream Taq DNA polymerase (Fermentas) and $1 \times$ Dream Taq buffer. The thermal profile was as follows: $94{ }^{\circ} \mathrm{C}(2 \mathrm{~min}), 30$ cycles of $94{ }^{\circ} \mathrm{C}(30 \mathrm{~s}), 50{ }^{\circ} \mathrm{C}(40 \mathrm{~s}), 68^{\circ} \mathrm{C}(1 \mathrm{~min})$ and $68^{\circ} \mathrm{C}$ ( $5 \mathrm{~min}$ ). PCR products were separated in $1 \%$ agarose gels, three products of expected size and all amplicons larger than expected were cut out, purified, cloned, and sequenced. Subsequently, PCR under the same conditions as above was carried out using DNAs of 141 randomly chosen BAC clones. Amplicons were separated in $1 \%$ agarose gels and scored. We estimated the copy number of DcSto 1 elements in the carrot genome taking into account that the average BAC clone size was $0.121 \mathrm{Mbp}$ (Cavagnaro et al. 2009) and that the $2 \mathrm{n}$ carrot genome was approximately $980 \mathrm{Mbp}$ (Bennett and Leith 1995).

Inverse PCR, Design, and Validation of Site-Specific Primers

Inverse PCR was set up as described by Collins and Weissman (1984). Ca. $100 \mathrm{ng}$ of genomic DNA was digested with $1 \mathrm{U}$ TaqI $\left(5^{\prime} \mathrm{T}^{\wedge} \mathrm{CGA} 3^{\prime}\right), M s p I\left(5^{\prime} \mathrm{C}^{\wedge} \mathrm{CGG} 3^{\prime}\right)$, EcoRI $\left(5^{\prime} \mathrm{G}^{\wedge} \mathrm{AATTC} 3^{\prime}\right)$, or $N d e \mathrm{I}\left(5^{\prime} \mathrm{CA}^{\wedge} \mathrm{TATG}^{\prime}\right)$ (Fermentas) at $37{ }^{\circ} \mathrm{C}$ for $3 \mathrm{~h}$ in the total volume of $10 \mu \mathrm{l}$ and incubated for $20 \mathrm{~min}$ at $65^{\circ} \mathrm{C}$ for thermal inactivation of the enzyme. Within DcSto sequence, restriction sites of the applied enzymes were not present. To achieve intramolecular circularization, $5 \mu \mathrm{l}$ of each digestion mixture was incubated overnight at $4{ }^{\circ} \mathrm{C}$ with $5 \mathrm{U}$ of T4 DNA ligase (Fermantas).

Inverse PCR products were amplified with primers anchored in opposite directions at both ends of the DcSto 1 element (DcSiPCR-F 5' CCA CTT CAC CCA CTT TTC CT $3^{\prime}$ and DcSiPCR-R 5'TTT TAG GAA AGT TTT GTA ATG TAA AGA $3^{\prime}$ ). Primers were designed with Primer3 (v. 0.4.0) (Rozen and Skaletsky 2000) based on the sequence of identified DcSto elements. Each $20 \mu \mathrm{l}$ reaction mixture contained $10 \mathrm{ng}$ self-ligated DNA, $1 \mathrm{mM}$ each primer, $0.25 \mathrm{mM}$ dNTPs (Fermentas), $1 \mathrm{U}$ Taq DNA polymerase (Fermentas) and $1 \times$ Taq buffer. The reactions were carried out as follows: $94{ }^{\circ} \mathrm{C}(1 \mathrm{~min}), 30$ cycles of $94{ }^{\circ} \mathrm{C}(30 \mathrm{~s}), 50{ }^{\circ} \mathrm{C}(45 \mathrm{~s}), 68^{\circ} \mathrm{C}(4 \mathrm{~min})$, and a final extension step of $68^{\circ} \mathrm{C}(10 \mathrm{~min})$. To obtain flanking sequences long enough to design site-specific primers, only products longer than $400 \mathrm{bp}$ were cloned and sequenced.

Local BLAST search was used to mine BAC-end sequences (BES) database with DcSto 1 sequence as a query (e-value cutoff was 0.01). Identified BES sequences with insertions of DcSto elements carrying characteristic TSD, TIR sequence, and for which enough flanking sequence was available, were used for further analysis. Boundary sequences of insertion sites obtained following iPCR or in silico analysis of BES, were used to design site-specific primers with Primer3 (v. 0.4.0) using default parameters.

Site-specific PCR was carried out in $10 \mu \mathrm{l}$ containing around $20 \mathrm{ng}$ genomic DNA, $1 \mathrm{mM}$ forward and reverse primer, $0.25 \mathrm{mM}$ dNTPs (Fermentas), $0.5 \mathrm{U}$ Taq DNA polymerase (Fermentas), and $1 \times$ Taq buffer supplied with $\mathrm{MgCl}_{2}$ (Fermentas). Amplification profile was as follows: $94{ }^{\circ} \mathrm{C}$ ( $\left.1 \mathrm{~min}\right), 30$ cycles of $94{ }^{\circ} \mathrm{C}$ (30 s), variable annealing temperature from 55 to $58{ }^{\circ} \mathrm{C}$ (depending on the primer combination) $(30 \mathrm{~s}), 68^{\circ} \mathrm{C}$ (variable time depending on the primer combination, from 1 to $3 \mathrm{~min}$ ), and $68^{\circ} \mathrm{C}$ (6 min). All primer sequences, the corresponding annealing temperatures and times of elongation are provided in supplementary Table 2 . Products were separated on $1 \%$ agarose gels and selected amplicons were sequenced.

Sequence evaluation and analysis

DcSto sequences were aligned using ClustalX (Thompson et al. 1997) and manually edited in BioEdit (Hall 1999). Genetic distances were calculated with Dnadist in PHYLIP (Felsenstein 1996) using Kimura two-parameter model of nucleotide substitution, Neighbor Joining (NJ) tree was produced with Neighbor and plotted with TreeView (Page 1996). To represent relationships among DcSto1 elements amplified from different sources, NJ tree was generated using MEGA 5.05 (Tamura et al. 2011). Consensus sequences of DcSto 1 to DcSto9 were used to predict secondary structures in RNAfold (Hofacker 2003), to search for putative promoter regions using TSSP (Softberry), $3^{\prime}$ end cleavage and polyadenylation sites using POLYAH (Softberry), regulatory DNA sequences in RegSite database using NSITE-PL (Softberry), and to identify transposons inserted in/close to coding regions in the sequences deposited in GenBank using blastn algorithm (Altschul et al. 1997). 
Fluorescence in situ hybridization

Localization of DcSto 1 elements on chromosomes of cv. Amsterdam 3 (AS33) was carried out by means of fluorescence in situ hybridization (FISH). The DcSto1 probe was amplified with DcS-TIR primer and cloned into pGEM-T vector. All steps of multi color FISH were carried out as described by Nowicka et al. (2012).

\section{Southern hybridization}

Digestion of $10 \mu \mathrm{g}$ genomic DNA was performed in $400 \mu \mathrm{l}$ with $100 \mathrm{U}$ of EcoRI (Fermentas) in $1 \times$ of EcoRI buffer for $3 \mathrm{~h}$ at $37{ }^{\circ} \mathrm{C}$ followed by $20 \mathrm{~min}$ at $65{ }^{\circ} \mathrm{C}$. Digested DNAs were separated in $1 \%$ TAE agarose gel for $16 \mathrm{~h}$ at $1 \mathrm{~V} / \mathrm{cm}$. The gel was washed for $8 \mathrm{~min}$ with $0.25 \mathrm{M} \mathrm{HCl}$, twice for $15 \mathrm{~min}$ with denaturation buffer $(0.5 \mathrm{M} \mathrm{NaOH}, 1.5 \mathrm{M}$ $\mathrm{NaCl}$ ), 1 min with distilled water, twice for 15 min with neutralization buffer (0.5 M Tris- $\mathrm{HCl}, \mathrm{pH} 7.5,1.5 \mathrm{M} \mathrm{NaCl})$. After washing with $20 \times \mathrm{SSC}(3 \mathrm{M} \mathrm{NaCl}, 0.3 \mathrm{M}$ sodium citrate, $\mathrm{pH} 7$ ) for $15 \mathrm{~min}$, the DNA was transferred onto Immobilion $^{\mathrm{TM}}$-P transfer membrane (Millipore) at room temperature. The membrane was then exposed to UV light (120 mJ/ $\mathrm{cm}^{2}$ ) using CL-100 Ultraviolet Crosslinker (UVP), washed for 2 min with $2 \times \mathrm{SSC}$, and dried. To prepare the probe, PCR was set up in $30 \mu \mathrm{l}$ using $15 \mathrm{ng}$ of template (a DcSto 1 element cloned into pGEM-T) with $1 \mathrm{mM} \mathrm{DcS-TIR}$ primer, $\quad 0.1 \mathrm{mM}$ digoxigenin-11-dUTP alkali-labile (Roche), $0.25 \mathrm{mM}$ dNTPs (Fermentas), 1.5 U Long PCR Enzyme Mix (Fermentas) and $1 \times$ long PCR buffer. The temperature profile was as follows: $94{ }^{\circ} \mathrm{C}(1 \mathrm{~min}), 30$ cycles of $94{ }^{\circ} \mathrm{C}(30 \mathrm{~s}), 50{ }^{\circ} \mathrm{C}(40 \mathrm{~s}), 68^{\circ} \mathrm{C}(1 \mathrm{~min})$ and $68{ }^{\circ} \mathrm{C}$ (4 min). Overnight hybridization at $65{ }^{\circ} \mathrm{C}$ was performed in $10 \mathrm{ml}$ of hybridization buffer $(7 \% \mathrm{SDS}, 50 \%$ deionized formamide, $5 \times \mathrm{SSC}, 0.1 \% \mathrm{~N}$-lauroyl sarcosine, $2 \%$ blocking solution, $50 \mathrm{mM}$ sodium phosphate, $\mathrm{pH}$ 7.0) with $30 \mu \mathrm{l}$ of the denatured probe. Detection was carried out using DIG luminescent detection kit (Roche) following instructions provided by the manufacturer.

\section{Results}

Identification and characteristics of DcSto elements

Upon a routine analysis of length polymorphism at the $r s$ locus (Yau et al. 2005) in carrot breeding materials of American origin we identified an undescribed 274 bp-long insertion characterized by a two-nucleotide 'TA' TSD and $16 \mathrm{bp}$-long TIRs. Based on the presence of TSD and TIR sequences, the inserted element was classified as a Stowaway-like MITE and named DcSto1-rs. Sequence of that element served as a starting point to identify more copies of DcSto. In total, we identified 89 elements using different methods (Table 1). On the basis of the commonly accepted 80-80-80 criterion (Wicker et al. 2007), the DcSto elements were divided into nine families, DcSto 1 to DcSto9. Grouping of twenty DcSto for which the full sequence was available (i.e. excluding those identified by amplification with the DcS-TIR primer) is in Fig. 1. Members of DcSto1, DcSto2, and DcSto3, were represented by more than one element in each category. Elements DcSto4, DcSto5, and DcSto6 were related to DcSto1, DcSto2, and DcSto3, respectively while DcSto7, DcSto8, and DcSto9 were more distant.

Among the DcSto elements identified, 73 copies represented DcSto1 and their average sequence similarity was $86 \%$. They could be grouped into branches on the Neighbor-Joining tree, however, the grouping did not

Table 1 Number of DcSto copies and methods used for their identification

\begin{tabular}{|c|c|c|c|c|c|c|}
\hline \multirow{2}{*}{$\begin{array}{l}\text { MITE } \\
\text { family }\end{array}$} & \multicolumn{6}{|c|}{ The method used for identification } \\
\hline & $\begin{array}{l}\text { TIR-PCR }{ }^{\mathrm{a}} \text { from } \\
\text { genomic DNA }\end{array}$ & $\begin{array}{l}\text { TIR-PCR }{ }^{\mathrm{a}} \text { from } \\
\text { BAC clones }^{\text {B }}\end{array}$ & iPCR & $\begin{array}{l}\text { BES in silico } \\
\text { analysis }\end{array}$ & $\begin{array}{l}\text { Site-specific } \\
\text { PCR }\end{array}$ & Reanalysis of published sequences \\
\hline DcSto 1 & 62 & 3 & 2 & 5 & - & - \\
\hline DcSto2 & - & $1^{\mathrm{b}}$ & 1 & 1 & 1 & - \\
\hline DcSto3 & 2 & 1 & - & 2 & 1 & - \\
\hline DcSto4 & - & - & - & - & 1 & - \\
\hline DcSto5 & - & - & - & - & 1 & - \\
\hline DcSto6 & - & - & - & - & 1 & - \\
\hline DcSto7 & - & - & - & - & - & $1($ AOX2a, GQ248714, Cardoso et al. 2009) \\
\hline DcSto8 & - & - & - & - & - & 1 ( $g$ DcPAL3, AB089813, Kimura et al. 2008) \\
\hline DcSto9 & - & - & - & - & - & $\begin{array}{l}1 \text { (lipid body membrane protein, S47635, } \\
\text { Hatzopoulos et al. 1990) }\end{array}$ \\
\hline
\end{tabular}

\footnotetext{
${ }^{a}$ Amplification with DcS-TIR primer specific to TIRs of DcSto 1
}

b Identified as nested insterion in DcSto1, see Fig. 1b 


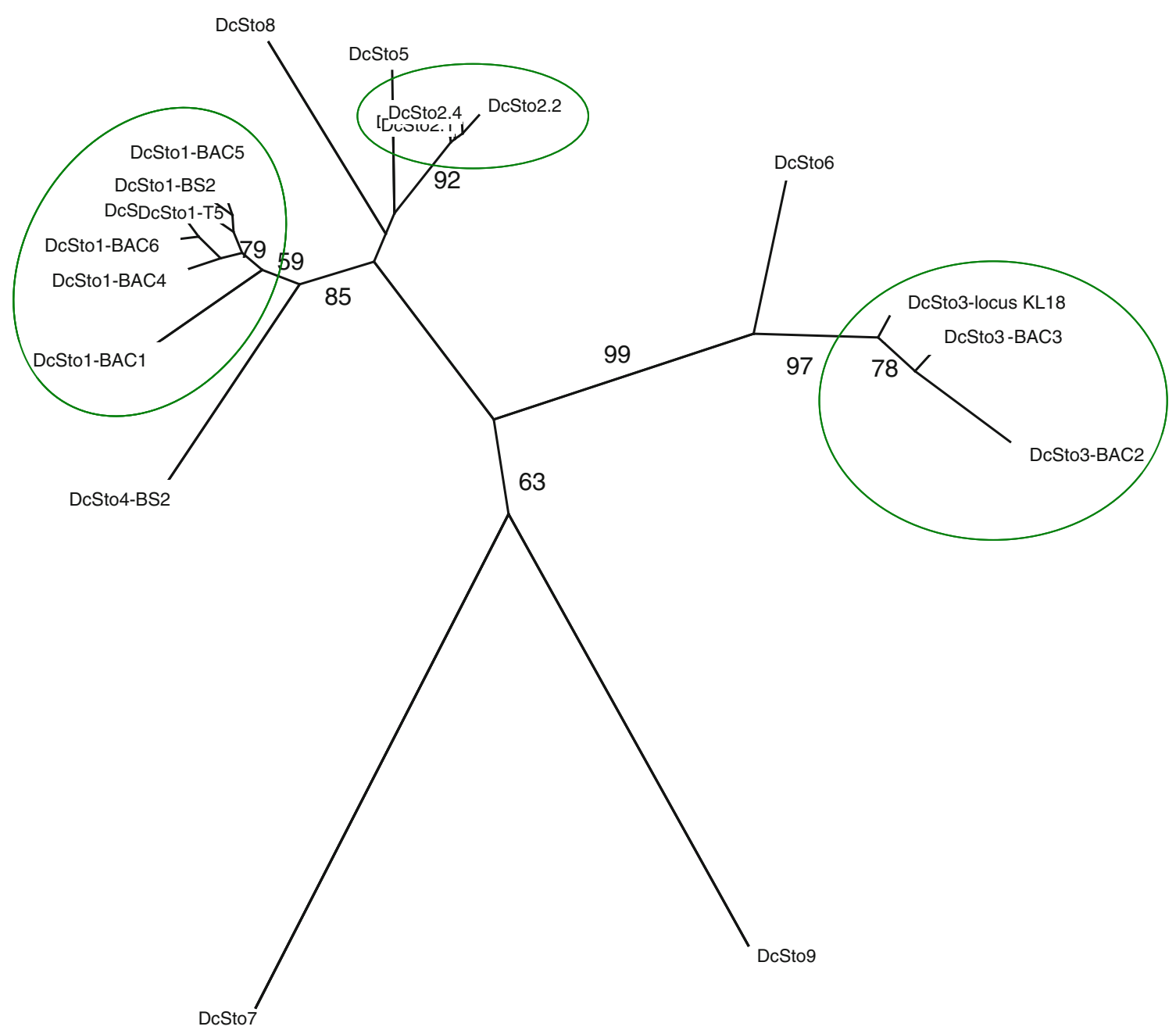

0.1

Fig. 1 A Neighbor-Joining tree showing relationships among carrot DcSto elements. Clusters comprising copies of DcSto1, DcSto2, and DcSto3 elements are circled

correspond with their origin from cultivated versus wild Daucus (Fig. 2). For DcSto2 and DcSto3, four and six copies, respectively, were observed and each of the remaining six families was represented by one element.

The TIR length varied from $13 \mathrm{bp}$ for DcSto4 to complete sequence folding into almost perfect hairpin structures in DcSto3, DcSto6, and DcSto9 (supplementary Figure 1). However, a highly conserved 6 bp-long terminal motif CTCCCT was always distinguishable (Fig. 3). As observed for other MITEs, the AT content of DcSto sequences was high (60-72\%).

Abundance and genomic distribution of DcSto 1

PCR amplification with the DcS-TIR primer was set up to screen 141 randomly chosen BAC clones. Amplicons of expected size were present in 87 of them. Thus, on average one DcSto 1 element was present per $196 \mathrm{~kb}$ and the copy number of DcSto 1 could be estimated as ca. 5,000 per diploid carrot genome. The high copy number of DcStol elements was confirmed by Southern hybridization with the DcSto 1 element used as a probe. Hybridization to EcoRI-digested DNA of cultivated and wild carrots resulted in a smear, indicating presence of DcSto1 in numerous copies (supplementary Figure 2). We also investigated the physical distribution of DcSto 1 along the carrot chromosomes using fluorescent in situ hybridization. DcSto 1 elements were present on both arms of all chromosomes but they were absent in the centromeric, telomeric, subtelomeric, and nucleolar organizer regions. In general, DcSto1 showed clustered distribution along the euchromatic regions of chromosome arms and their co-localization with the DAPI stained blocks of heterochromatin was not observed (Fig. 4). 
Fig. 2 A Neighbor-Joining tree showing relationships among DcSto 1 elements originating from different Daucus host genomes. DcSto1-rs is labeled red, DcSto elements amplified from cultivated carrot are labeled green and those from wild Daucus (sub)species are labeled blue. (Color figure online)
Fig. 3 Alignment of TIR sequences of DcSto families (Y stands for $\mathrm{T} / \mathrm{C}, \mathrm{W}$ for $\mathrm{A} / \mathrm{T}, \mathrm{S}$ for $\mathrm{C} / \mathrm{G}, \mathrm{R}$ for $\mathrm{A} / \mathrm{G}$ and $\mathrm{M}$ for $\mathrm{A} / \mathrm{C}$ ). The red rectangle indicates the conserved 6 nt-long TIR portion. (Color figure online)

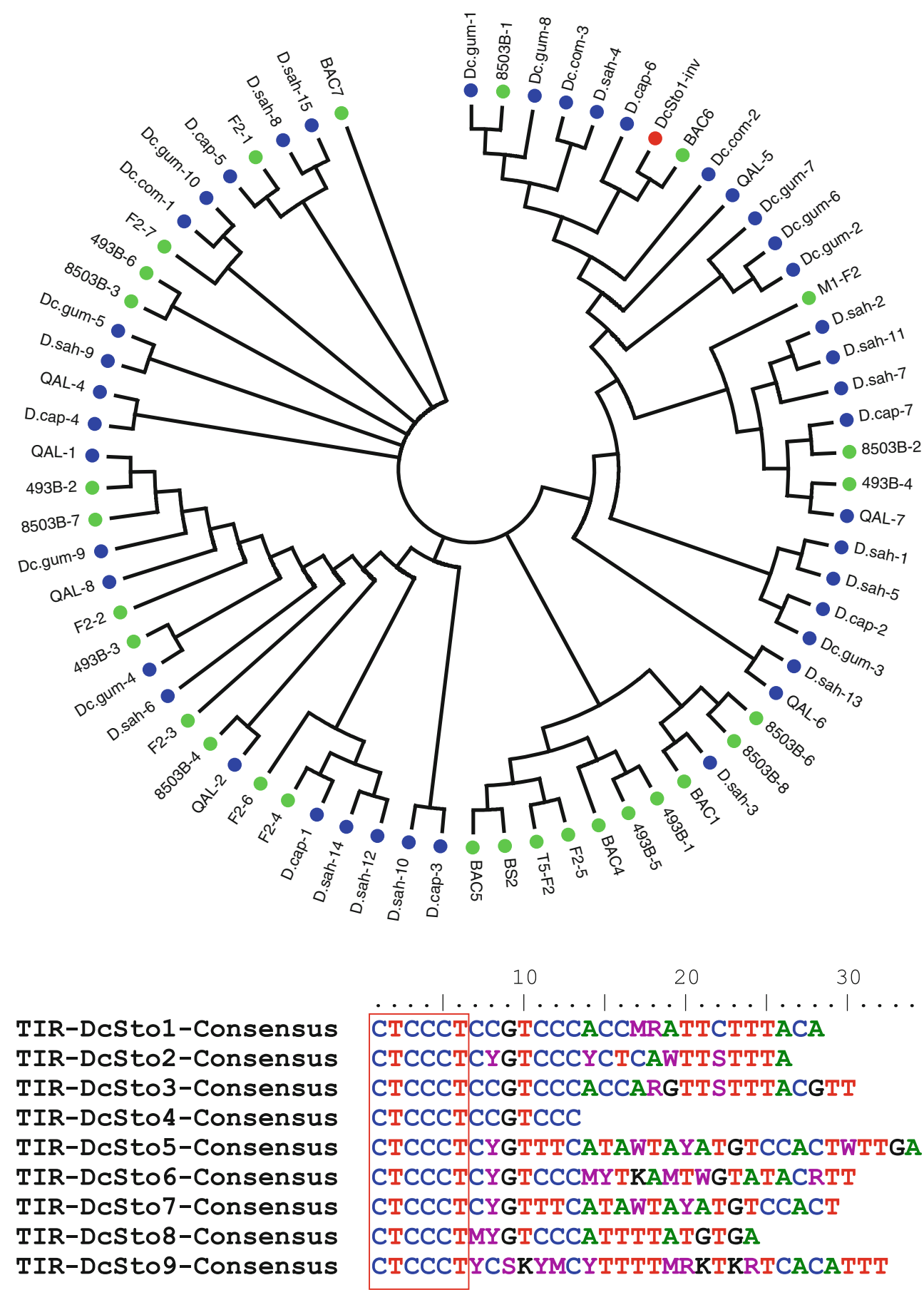

Local rearrangements resulting from the activity of DcSto elements

PCR amplification of DcSto transposons from carrot BAC clones using DcS-TIR primer produced three bands of sizes longer than expected for DcSto1. One of them was a DcSto 1 element carrying additional $59 \mathrm{nt}$ similar to the terminal part of the transposon at the $5^{\prime}$ end (Fig. 5b). Those extra nucleotides, excluding the sequence of the TIR-primer, were more similar to other DcSto copies then to the adjacent element, suggesting a nested insertion into another DcSto element. Two other products were derived from DcSto1 carrying nested insertions of a complete DcSto2 element or an unrelated Tourist-like MITE (Fig. 5c, d).

To characterize local variation in DcSto insertion sites, primers flanking DcSto insertions identified in BES were used to re-amplify eight loci in unrelated individuals. All insertion sites were polymorphic among analyzed plants of cultivated carrot. 

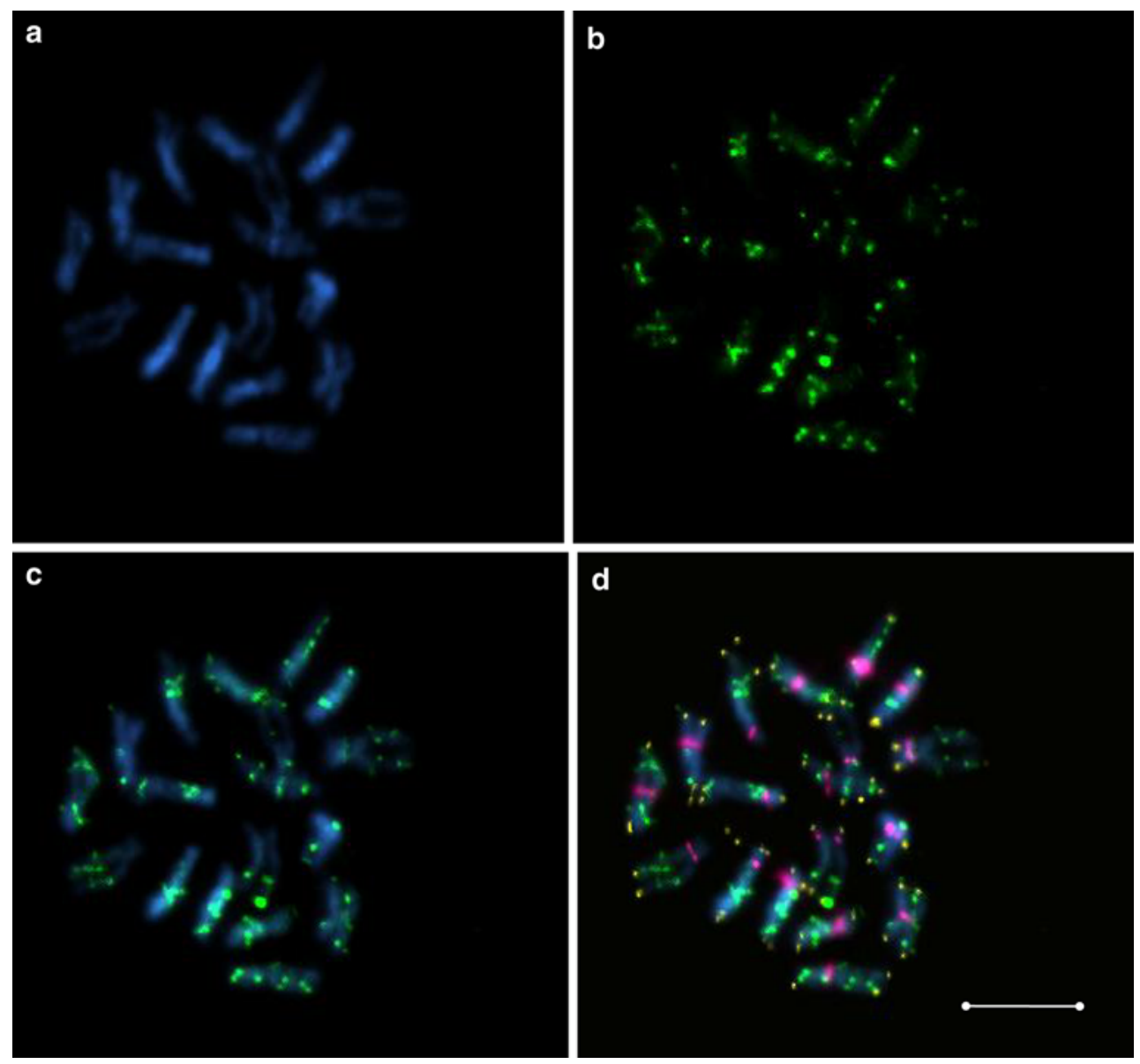

Fig. 4 Localization of DcSto 1 elements along carrot cv. Amsterdam 3 (AS33) chromosomes by multi-color fluorescence in situ hybridization (a-d). DAPI-stained mitotic chromosomes (blue) (a); distribution of the DcSto1 probe detected with FITC-conjugated antidigoxigenin antibody (green) (b); distribution of the DcSto1 probe detected with FITC-conjugated anti-digoxigenin antibody (green) on

In case of three loci, besides expected size variants representing empty/occupied site, more complex rearrangements were also observed. In the BS2.1 region, a complete DcSto1 was identified, but also two of its derivatives, likely resulting from abortive gap repair following an excision event. Additionally, one of the derived DcSto 1 variants was accompanied by an insertion of a DcSto6 element 20 bp upstream the DcSto1 insertion (Fig. 6). We have not identified a variant carrying a solo insertion of DcSto6, which suggests that the latter element was inserted into the BS2.1 variant with the internally truncated DcSto 1 element already present. The BS2.2 region was characterized by alternative insertions of two different DcSto elements, DcSto3 and DcSto4, in exactly the same position. Also, a short deletion around the insertion site, likely resulting from an excision event, was observed in one of the variants (supplementary Figure 3). In the BS4 region, three independent insertions of DcSto1, DcSto5, and an uncharacterized 549 bp-long indel were found in different genetic

DAPI-stained chromosomes (blue) (c); overlapped hybridization signals of the DcSto1 probe (green), the centromeric probe [Cy5, red signal was changed to purple with Case Data Manager (ASI)] and the telomeric probe [Cy3, red signal was changed to yellow with Case Data Manager (ASI)] on the same metaphase stained with DAPI (blue) $(\mathbf{d})$. Scale bar $=5 \mu \mathrm{m}$. (Color figure online)

backgrounds (supplementary Figure 4). DcSto5 was inserted 48 bp upstream the DcSto 1 insertion site and a variant carrying insertions of both elements was not identified.

The presence of non-fixed DcSto insertions in Daucus carota suggests an extensive recent transpositional activity of those elements. One particularly interesting case of DcSto mobilization came from an analysis of size polymorphism in the first intron of the carrot chxbl gene. A longer version of the intron, resulting from the insertion of a DcSto2 element was present and segregating only in AS38, one of more than 160 screened accessions (supplementary Figure 5), indicating a very recent insertion event limited to a single population of cultivated carrots.

Presence of DcSto elements in the vicinity of coding regions

DcSto elements were identified upon examination of published sequences in the vicinity of genes of Apioideae 
a

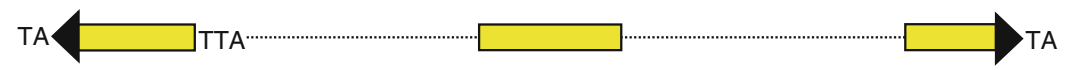

b

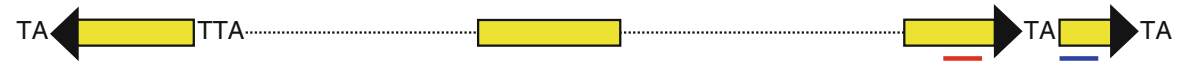

C

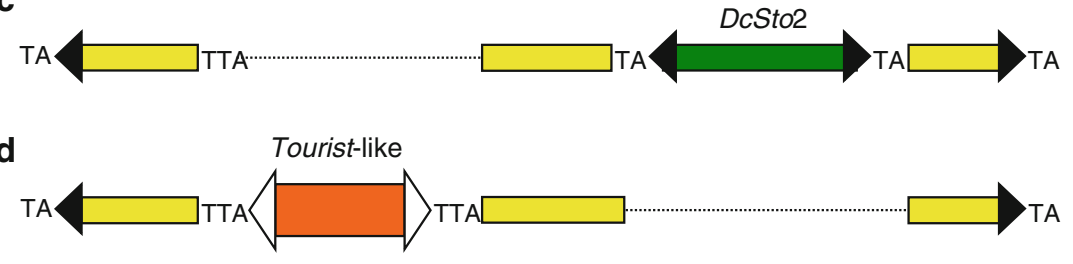

DcSto TIR $\$ Tourist-like MITE TIR

Fig. 5 Graphic representation of nested insertions identified in PCR fragments generated with DcS-TIR primer anchored in DcSto1 TIRs. A complete DcSto 1 sequence (a), a duplicated terminal DcSto sequence, red line indicates the region of DcSto with $93 \%$ similarity to DcSto-rs and blue line indicates the region of DcSto with $73 \%$ similarity to DcSto-rs (b), nested insertion of DcSto2 (c), nested insertion of a Tourist-like MITE (d), not drawn to scale. Target site duplications are marked as TA (Stowaway type) and TTA (Tourist type), dotted lines represent gaps, yellow bars represent DcSto1, orange and green bars represent nested insertions of Tourist-like MITE and DcSto2, respectively. (Color figure online)
Fig. 6 Schematic

representation of structural variants identified in BS2.1 locus (a) and amplification profiles for a collection of plants representing diversity of the cultivated carrot (b). Yellow and red bars indicate insertions of DcSto 1 and DcSto6 elements, respectively. (Color figure online)

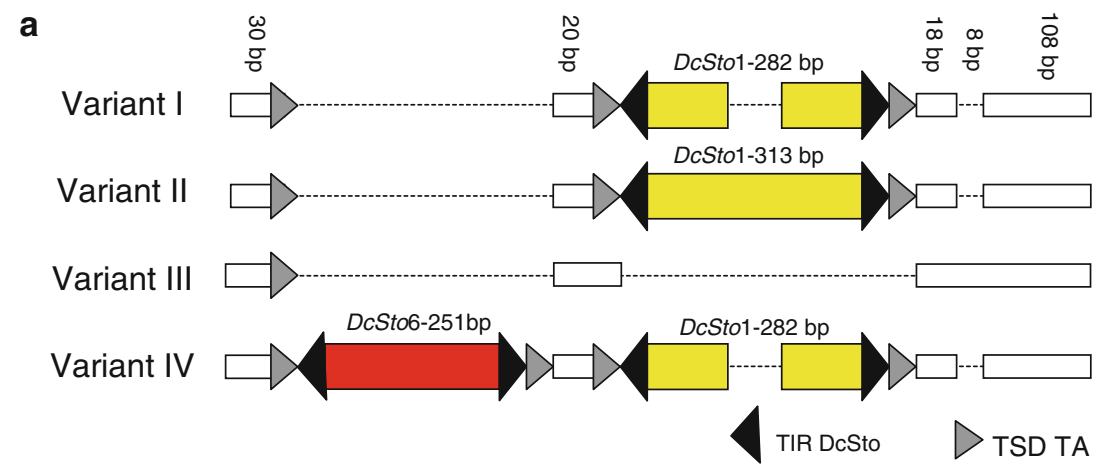

b

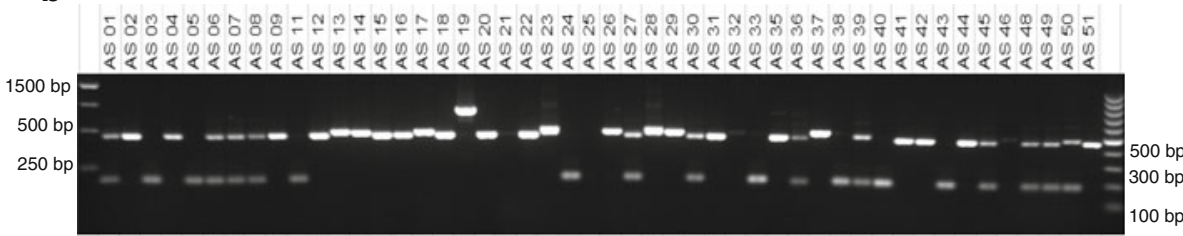

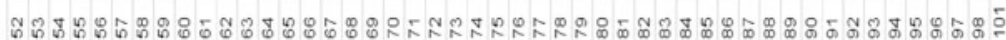

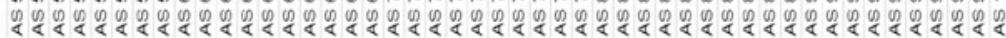

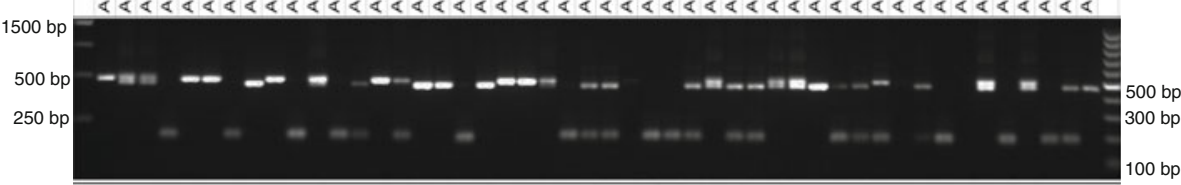

(carrot, Petroselinum crispum, and Bupleurum kaoi), especially within $5^{\prime}$ UTR regions and upstream of transcription start sites (Table 2). Complete elements were identified upstream of two carrot genes, i.e. $g D c P A L 3$, and $I n v^{*} D c 5$. Interestingly, other DcSto copies associated with genic regions were devoid of TIR and the typical TSD at one or both ends, thus they were immobilized.

DcSto6 showed $70 \%$ similarity over the entire element to the complete Stowaway-like MITE in parsley, which we named PcSto (Petroselinum crispum Stowaway), identified 
Table 2 Regions carrying insertions of DcSto-like elements close to or in coding sequences of Apioideae

\begin{tabular}{|c|c|c|c|}
\hline $\begin{array}{l}\text { Accession } \\
\text { number }\end{array}$ & Gene & DcSto characteristics & Localization \\
\hline \multicolumn{4}{|l|}{ DcSto 1} \\
\hline AB050962 & Daucus carota DcECP40 gene for ECP40, promoter region & Lack of two TIRs & Promoter region \\
\hline AJ303199 & Daucus carota sut 2 gene for sucrose/proton symporter, exons $1-4$ & Lack of one TIR & Intron \\
\hline \multicolumn{4}{|l|}{ DcSto3 } \\
\hline AB118876 & Daucus carota $C-A B I 3$ gene, upstream region & Lack of one TIR & Promoter region \\
\hline \multicolumn{4}{|l|}{ DcSto5 } \\
\hline AB089813 & Daucus carota gDcPAL3 gene for phenylalanine ammonia-lyase & Complete & $1,933 \mathrm{bp}$ upstream of cds \\
\hline \multicolumn{4}{|l|}{ DcSto6 } \\
\hline X16131 & D. carota $D C 8$ gene for an embryonic-specific $66 \mathrm{kDa}$ protein & Lack of one TIR & $761 \mathrm{bp}$ upstream of cds \\
\hline U46217 & $\begin{array}{l}\text { Petroselinum crispum common plant regulatory factor } C P R F 1 \text { gene, } \\
\text { promoter region and complete cds }\end{array}$ & Complete & Intron \\
\hline X55736 & Petroselinum crispum $P R 2$ gene for pathogenesis-related protein 2 & Complete & Promoter region \\
\hline AF274564 & $\begin{array}{l}\text { Petroselinum crispum immediate-early fungal elicitor protein } \\
\text { CMPG1 } \\
\text { gene, partial cds }\end{array}$ & Complete & $1,391 \mathrm{bp}$ upstream of cds \\
\hline AF121354 & Petroselinum crispum transcription factor $W R K Y 3$ gene, partial cds & Complete & $3,417 \mathrm{bp}$ upstream of cds \\
\hline AF239835 & $\begin{array}{l}\text { Petroselinum crispum fatty acid desaturase/hydroxylase-like protein } \\
\text { ELI7.1 gene, complete cds; and fatty acid desaturase/hydroxylase- } \\
\text { like protein ELI7.2 gene, partial }\end{array}$ & Partial & $\begin{array}{l}\text { Between two predicted } \\
\text { cds (ELI7.1 and ELI7.2) }\end{array}$ \\
\hline Y16091 & Daucus carota Susy*Dc2 gene & Lack of one TIR & $3^{\prime}$ UTR \\
\hline Y18706 & Daucus carota vacuolar invertase isoenzyme II gene (Inv*Dc5) & Complete & $5^{\prime}$ UTR \\
\hline \multicolumn{4}{|l|}{ DcSto7 } \\
\hline AB188289 & $\begin{array}{l}\text { Daucus carota } C \text {-EF1 mRNA for embryonic element binding } \\
\text { Factor } 1 \text {, partial cds }\end{array}$ & Partial & $3^{\prime}$ UTR \\
\hline \multicolumn{4}{|l|}{ DcSto8 } \\
\hline FN667831 & Bupleurum kaoi mRNA for ethylene response factor 1 (erfl gene) & Partial & $3^{\prime} \mathrm{UTR}$ \\
\hline
\end{tabular}

in a region upstream of four $P$. crispum genes and in an intron of one gene (Table 2). An average sequence similarity of the four identified PcSto elements was $74 \%$, and only PcSto-PR2 and PcSto-CMPG1 were over $80 \%$ similar. Interestingly, DcSto6 and DcSto3 were more similar to the PcSto elements than to other carrot DcSto elements (supplementary Figure 6).

DcSto elements belonging to five families carried putative promoters and TATA boxes, while four families might provide polyA sites for adjacent genes. Also, within sequences of any DcSto family at least 5 putative regulatory motifs were present and only three families i.e. DcSto5, DcSto7, and DcSto8 carried less then ten putative regulatory motifs (Table 3 ).

\section{Discussion}

The present study demonstrated that DcSto MITEs were abundant and diverse in the carrot genome. We investigated the distribution of DcSto1 elements across cultivated and wild carrot, as well as two closely related species $D$. capillifolius and D. sahariensis. We did not observe any DcSto 1 lineages differentiating investigated groups of accessions, which might reflect their previously documented intra- and interspecific crossability (Grzebelus et al. 2011). We showed that DcSto elements in carrot were present in thousands of copies, which stays in agreement with the general characteristics of Stowaway elements present in other plants. For example, 18 Stowaway-like MITE families present in over 18,000 copies, were described in the wheat genome (Yaakov et al. 2013). Also, analysis of Stowaway elements in the relatively small genome of rice revealed presence of over 22,000 Stowaway elements divided into 36 families (Feschotte et al. 2003).

The high level of DcSto insertion polymorphism and low frequency of carrot plants harboring insertions of DcSto 1 into rs and DcSto 2 into chxbl genes suggests recent mobilization of DcSto elements. Insertion and excision events resulted in high local variability, including deletions of sequences surrounding the excision site, which was reported previously in rice (Yang et al. 2006, 2009). 
Table 3 Coordinates of putative promoters, regulatory motifs, and polyA signals within consensus DcSto sequences

\begin{tabular}{lllll}
\hline Family & $\begin{array}{l}\text { Position/strand of the } \\
\text { first nt of predicted } \\
\text { promoters }\end{array}$ & $\begin{array}{l}\text { Number/strand of } \\
\text { predicted transcription } \\
\text { factor binding sites }\end{array}$ & $\begin{array}{l}\text { Number/strand of } \\
\text { regulatory motifs }\end{array}$ & $\begin{array}{l}\text { Number/position/ } \\
\text { strand of predicted } \\
\text { polyA signals }\end{array}$ \\
\hline DcSto1 & $189 /+$ & $39 / 12(+), 27(-)$ & $21 / 13(+), 8(-)$ & 0 \\
DcSto2 & $167 /-$ & $15 / 7(+), 8(-)$ & $12 / 8(+), 4(-)$ & $1 / 137 /-$ \\
DcSto3 & - & 0 & $10 / 5(+), 5(-)$ & $1 / 104 /+$ \\
DcSto4 & $130 /+$ & $12 / 4(+), 8(-)$ & $12 / 4(+), 8(-)$ & 0 \\
DcSto5 & - & 0 & $5 / 3(+), 2(-)$ & 0 \\
DcSto6 & $142 /+$ & $30 / 12(+), 18(-)$ & $14 / 7(+), 7(-)$ & $1 / 112 /+$ \\
DcSto7 & - & 0 & $5 / 2(+), 3(-)$ & 0 \\
DcSto8 & $227 /+$ & $32 / 8(+), 24(-)$ & $7 / 1(+), 6(-)$ & $2 / 141,164 /+,-$ \\
DcSto9 & - & 0 & $16 / 5(+), 11(-)$ & 0 \\
\hline
\end{tabular}

We speculate that the DcSto3 and DcSto6 families were vertically inherited from the common ancestor by the Daucus and Petroselinum linkages as suggested by their high similarity to PcSto identified in the P. crispum genome. Despite the apparent long evolutionary history of these families, indicated by their likely presence in the genome of a common ancestor of Daucus and Petroselinum, a recent mobilization event of DcSto6 in the BS2.1 region was documented. As Stowaway MITEs were shown to be evolutionarily related to autonomous elements from the Tc1/mariner superfamily (Turcotte et al. 2001; Menzel et al. 2006), it has been commonly accepted that their mobilization relies on the availability of mariner transposases (Feschotte et al. 2003). However, autonomous elements serving as transposase donors for DcSto elements remain to be identified. As proposed by Jiang et al. (2004), MITE precursors originated from autonomous elements, but their proliferation was driven later by transposases encoded by autonomous elements, not directly related but still capable of recognizing MITE termini. Also, relatively few changes in TIRs may have a dramatic effect on transposase binding to the element ends (Lampe et al. 2001). According to this scenario, divergence of TIRs sequences of DcSto elements may have resulted in mobilization of a particular group by different mariner-like transposases within overlapping time-frames. A hypothesis of multiple bursts of transposition was also proposed by Grzebelus et al. (2009) to explain diversity and evolutionary history of Medicago PIF/Harbinger-related MITEs.

We found evidence for clustered insertions of DcSto. FISH with DcSto1 revealed clustered signals over all carrot chromosomes. Analysis of the local structure of carrot DcSto insertion sites revealed clustered insertions (BS2.1) and independent insertions of DcSto into the same position or very close to the insertion site of other elements in plants of different origin (BS2.2, BS4). Analysis of maize and rice
MITEs insertion site preference shows that insertion of MITEs into other members of the same family were common. It was proposed that nested and clustered insertions may act as a mechanism of limitation of transposition frequency and 'safe haven' where further integration of transposons would be tolerated (Rothnie et al. 1990; Jiang and Wessler 2001).

Independent insertions of Stowaway elements into the same localization were shown in rice $\mathrm{R}$ genes $(\mathrm{Hu}$ et al. 2000) and Triticaeae $\beta$-amylase gene (Mason-Gamer 2007). In addition, Stowaways were identified in maize $b z$ locus, referred to as a transpositional 'hot spot' and characterized by multiple insertions of MITEs, DNA transposons, and retrotransposons (Wang and Dooner 2006). The first DcSto element was found in the first intron of carrot soluble invertase isozyme II ( $r s)$. Interestingly, the same intron was previously reported as harboring insertion of a non-autonomous PIF/Harbinger element DcMaster 1 (Grzebelus et al. 2006) which might suggest that the intron acted as a similar transpositional 'hot spot'.

As observed for other MITEs, DcSto elements were frequently inserted in the vicinity of genes (Mao et al. 2000, Yaakov et al. 2013). Besides the $r s$ gene, we found a copy of DcSto in the first intron of the chxbl gene and reanalyzed previous reports on insertions in or near carrot genes. Cardoso et al. (2009) identified an indel in the third intron of the $A O X 2 a$ gene which we found to be a DcSto. Kimura et al. (2008) described an insertion of a $299 \mathrm{bp}$ MITE in the promoter region of the phenylalanine ammonia-lyase gene (DcPAL3) close to another MITE element. The former MITE could be attributed to the DcSto group. Also, we identified a DcSto element inserted in the region upstream carrot lipid body membrane protein gene (Hatzopoulos et al. 1990).

Presence of putative promoters and polyA motifs within sequence of most of analyzed DcSto elements and presence 
of sequences constituting putative regulatory elements for all families might indicate their possible influence on the expression of adjacent genes. The effect of DcSto 8 on the expression of carrot phenylalanine ammonia-lyase gene (DcPAL3) analyzed by Kimura et al. (2008), showed that clustered insertion of DcSto8 and another MITE significantly increased the transcription level. That effect may be one of the reasons for a relatively frequent occurrence of DcSto elements in clusters with other MITEs observed previously and confirmed in the present study. Analysis of the binding capacity of a region upstream a lipid body membrane protein gene from carrot overlapping with the DcSto9 insertion showed that the region had a potential to form complexes with nuclear extracts from embryos (Hatzopoulos et al. 1990). Interestingly, the region identified as responsible for DNA-binding, harbored by DcSto9, was not present in other DcSto element families.

Very recently, it was shown that more than half of miRNAs associated with rice transposable elements originated from MITEs (Yu et al. 2010, Sanan-Mishra et al. 2009). Similar results were observed in Solanaceae (Kuang et al. 2009) and Arabidopsis (Hollister et al. 2011). Readthrough transcription of MITEs inserted into UTR regions of genes may lead to their folding into hairpin structures, which are further processed into small RNAs. As shown in Arabidopsis, such MITE-derived miRNAs have a significant impact on the decrease of expression of adjacent genes, owing to higher methylation of those regions (Hollister et al. 2011). A predicted miRNA encoded by DcSto7, as proposed by Cardoso et al. (2009), showed significant similarity to a rice miRNA. Moreover, DcSto3 and DcSto6 copies, capable of forming fold-back structures, were most frequently associated with carrot transcripts (Iorizzo et al. 2011). Also, DcSto6 lacking one of the TIRs is present in the region upstream the carrot $D c 8$ gene showing differential expression during embryo development related to changes in the methylation pattern of the promoter region (Zhou et al. 1998). Also, DcSto6 elements shared $69 \%$ similarity over their entire sequence with PcSto elements, identified adjacent to coding regions of Petroselinum crispum, a related Apiaceae species. In addition, DcSto and related elements in Apioideae species identified in the vicinity of coding regions were frequently characterized by the lack of one or both TIRs. Loss of functional termini prevents mobilization of DcSto elements which might suggest their retention due to a putative adaptive effect on the expression of adjacent genes. We conclude that abundance of DcSto elements in euchromatic regions, their presence in carrot transcripts, and presence of putative regulatory motifs within their sequences may indicate their involvement in the regulation of gene expression.
Acknowledgments This research was financially supported by the Polish Ministry of Science and Higher Education, project DS3500/ KGHN granted to the University of Agriculture in Krakow.

Open Access This article is distributed under the terms of the Creative Commons Attribution License which permits any use, distribution, and reproduction in any medium, provided the original author(s) and the source are credited.

\section{References}

Altschul SF, Madden TL, Schäffer AA, Zhang J, Zhang Z, Miller W, Lipman DJ (1997) Gapped BLAST and PSI-BLAST: a new generation of protein database search programs. Nucl Acids Res 25:3389-3402

Arabidopsis Genome Initiative (2000) Analysis of the genome sequence of flowering plant Arabidopsis thaliana. Nature 408: 796-815

Baranski R, Maksylewicz-Kaul A, Nothnagel T, Cavagnaro P, Simon PW, Grzebelus D (2011) Genetic diversity of carrot (Daucus catota L.) cultivars revealed by analysis of SSr loci. Genet Resour Crop Evol 59:163-170

Bennett MD, Leith IJ (1995) Nuclear DNA amounts in angiosperms. Ann Bot 76:113-176

Bureau TE, Wessler SR (1994) Stowaway: a new family of inverted repeat elements associated with the genes of both monocotyledonous and dicotyledonous plants. Plant Cell 6:907-916

Cardoso HG, Campos MD, Costa AR, Campos MC, Nothnagel T, Arnholdt-Schmitt B (2009) Carrot alternative oxidase gene AOX2a demonstrates allelic and genotypic polymorphisms in intron 3. Physiol Plantarum 137:592-608

Cavagnaro PF, Chung SM, Szklarczyk M, Grzebelus D, Senalik D, Atkins AE, Simon PW (2009) Characterization of a deepcoverage carrot (Daucus carota L.) BAC library and initial analysis of BAC-end sequences. Mol Genet Genomics 281: 273-288

Collins FS, Weissman SM (1984) Directional cloning of DNA fragments at large distance from an initial probe: a circularization method. Proc Natl Acad Sci USA 81:6812-6816

Felsenstein J (1996) Inferring phylogenies from protein sequences by parsimony, distance, and likelihood methods. Methods Enzymol 266:418-427

Feschotte C, Wessler SR (2002) Mariner-like transposases are widespread and diverse in flowering plants. Proc Natl Acad Sci USA 99:280-285

Feschotte C, Swamy L, Wessler SR (2003) Genome-wide analysis of Mariner-like transposable elements in rice reveals complex relationships with Stowaway miniature inverted repeat transposable elements (MITEs). Genetics 163:747-758

Feschotte C, Osterlund MT, Peeler R, Wessler SR (2005) DNAbinding specificity of rice mariner-like transposases and interactions with Stowaway MITEs. Nucl Acids Res 33:2153-2165

Finnegan DJ (1989) Eukaryotic transposable elements and genome evolution. Trends Genet 5:103-107

Grzebelus D, Simon PW (2009) Diversity of DcMaster-like elements of the PIF/Harbinger superfamily in the carrot genome. Genetica 135:347-353

Grzebelus D, Yau YY, Simon PW (2006) Master: a novel family of PIF/Harbinger-like transposable elements identified in carrot (Daucus carota L.). Mol Genet Genomics 275:450-459

Grzebelus D, Jagosz B, Simon PW (2007) The DcMaster transposon display maps polymorphic insertion sites in the carrot (Daucus carota L.) genome. Gene 390:67-74 
Grzebelus D, Gladysz M, Macko-Podgorni A, Gambin T, Golis B, Rakoczy R, Gambin A (2009) Population dynamics of miniature inverted-repeat transposable elements (MITEs) in Medicago truncatula. Gene 448:214-220

Grzebelus D, Baranski R, Spalik K, Allender C, Simon PW (2011) Daucus. In: Kole C (ed) Wild crop relatives: genomic and breeding resources. Vegetables. Springer, Berlin, pp 91-113

Hall TA (1999) Bioedit: a user-friendly biological sequence alignment editor and analysis program for Windows 95/98/NT. Nucl Acids Symp 41:95-98

Hatzopoulos P, Franz G, Choy L, Sung R (1990) Interaction of nuclear factors with upstream sequences of a lipid body membrane protein gene from carrot. Plant Cell 2:457-467

Hofacker SH (2003) Vienna RNA secondary structure server. Nucl Acids Res 31:3429-3431

Hollister JD, Smith LM, Guo YL, Ott F, Weigel D, Gaut BS (2011) Transposable elements and small RNAs contribute to gene expression divergence between Arabidopsis thaliana and Arabidopsis lyrata. Proc Natl Acad Sci USA 108:2322-2327

Hu J, Reddy VS, Wessler SW (2000) The rice R family: two distinct subfamilies containing several miniature inverted-repeat transposable elements. Plant Mol Biol 42:667-678

Iorizzo M, Senalik D, Grzebelus D, Bowman M, Cavagnaro PF, Ashrafi H, Van Deynze A, Simon PW (2011) De novo assembly of the carrot transcriptome from short-read sequences to characterize the genome and develop molecular markers. BMC Genomics 12:389

Itoh Y, Hasebe M, Davies E, Takeda J, Ozeki Y (2003) Survival of $T d c$ transposable elements of the En/Spm superfamily in the carrot genome. Mol Gen Genomics 269:49-59

Jiang N, Wessler SR (2001) Insertional preference of maize and rice miniature inverted repeat transposable elements as revealed by the analysis of nested elements. Plant Cell 13:2553-2564

Jiang N, Feschotte C, Zhang X, Wessler SR (2004) Using rice to understand the origin and amplification of miniature inverted repeat transposable elements (MITEs). Curr Opin Plant Biol 7:115-119

Kimura S, Mikiko O, Fukuda T, Ohno Y, Hongo C, Itoh Y, Koda T, Ozeki Y (2008) Role of miniature inverted repeat transposable elements inserted into the promoted region of carrot phenylalanine ammonia-lyase gene and its gene expression. Plant Biotechnol 25:473-481

Kuang H, Padmanabhan C, Li F, Kamei A, Bhaskar B, Ouyang S, Jiang J, Buell CR, Baker B (2009) Identification of miniature inverted-repeat transposable elements (MITEs) and biogenesis of their siRNA in the Solanaceae: new functional implications for MITEs. Genome Res 19:42-56

Lampe DJ, Churchill MEA, Robertson HM (1996) A purified mariner transposase is sufficient to mediate transposition in vitro. EMBO J 15:5470-5479

Lampe DJ, Walden KKO, Robertson HM (2001) Loss of transposaseDNA interaction may underlie the divergence of mariner family transposable elements and the ability of more than one mariner to occupy the same genome. Mol Biol Evol 18:954-961

Macas J, Koblizkowa A, Neumann P (2005) Characterization of Stowaway MITEs in pea (Pisum sativum L.) and identification of their potential master elements. Genome 48:831-839

Mao L, Wood TC, Yu Y, Budiman MA, Tomkins J, Woo SS, Sasinowski M, Presting G, Frish D, Goff S, Dean RA, Wing RA (2000) Rice transposable elements: a survey of 73,000 sequencetagged-connectors. Genome Res 10:982-990

Mason-Gamer RJ (2007) Multiple homoplasious insertions and deletions of a Triticae (Poaceae) DNA transposon: a phylogenetic perspective. BMC Evol Biol 7:92

Menzel G, Dechyeva D, Keller H, Lange C, Himmelbauer H, Schmidt $\mathrm{T}$ (2006) Mobilization and evolutionary history of miniature inverted-repeat transposable elements (MITEs) in Beta vulgaris L. Chromosom Res 14:831-844

Momose M, Abe Y, Ozeki Y (2010) Miniature inverted-repeat transposable elements of Stowaway are active in potato. Genetics 186:59-66

Nowicka A, Grzebelus E, Grzebelus D (2012) Fluorescent in situ hybridization with arbitrarily amplified DNA fragments differentiates carrot (Daucus carota L.) chromosomes. Genome 55: 205-213

Ozeki Y, Davies E, Takeda J (1997) Somatic variation during long term subculturing of plant cells caused by insertion of a transposable element in a phenylalanine ammonia-lyase (PAL) gene. Mol Gen Genet 254:407-416

Page RDM (1996) Treeview: an application to display phylogenetic trees on personal computers. Comput Appl Biosci 12:357-358

Rothnie HM, McCurrach KJ, Glover LA, Hardman N (1990) Retrotransposon-like nature of $T p 1$ elements: implications for the organization of highly repetitive, hypermethylated DNA in the genome of Physarum polucephalum. Nucleic Acids Res 19:279-286

Rozen S, Skaletsky HJ (2000) Primer3 on the WWW for general users and for biologist programmers. In: Krawetz S, Misener S (eds) Bioinformatics methods and protocols: methods in molecular biology. Humana Press, Totowa, NJ, pp 365-386, Source code available at http://fokker.wi.mit.edu/primer3/

Sanan-Mishra N, Kumar V, Sopory SK, Mukherjee SK (2009) Cloning and validation of novel miRNA from basmati rice indicates cross talk between abiotic and biotic stress. Mol Genet Genomics 282:463-474

Schnable PS et al (2009) The B73 maize genome: complexity, diversity, and dynamics. Science 326:1112-1115

Takata M, Kiyohara A, Takasu A, Kishima Y, Ohtsubo H, Sano Y (2007) Rice transposable elements are characterized by various methylation environments in the genome. BMC Genomics 8: 469

Tamura K, Peterson D, Peterson N, Stecher G, Nei M, Kumar S (2011) MEGA5: molecular evolutionary genetics analysis using maximum likelihood, evolutionary distance, and maximum parsimony methods. Mol Biol Evol 28:2731-2739

Thompson JD, Gibson TJ, Plewniak F, Jeanmougin F, Higgins DG (1997) The ClustalX windows interface: flexible strategies for multiple sequence alignment aided by quality analysis tool. Nucleic Acids Res 24:4876-4882

Turcotte K, Srinivasan S, Bureau TE (2001) Survey of transposable elements from rice genomic sequences. Plant J 25:169-179

Wang Q, Dooner HK (2006) Remarkable variation in maize genome structure inferred from haplotype diversity at $b z$ locus. Proc Natl Acad Sci USA 21:17644-17694

Wicker T, Sabot F, Hua-Van A, Bennetzen JL, Capy P, Chalhoub B, Flavell A, Leroy P, Morgante M, Panaud O, Paux E, SanMiguel P, Schulman AH (2007) A unified classification system for eukaryotic transposable elements. Nature Rev Genet 8: 973-982

Yaakov B, Ben-David S, Kashkush K (2013) Genome-wide analysis of Stowaway-like MITEs in wheat revealed high sequence conservation, association with genes and genomic diversification. Plant Physiol 161:486-496

Yang G, Weil CF, Wessler SR (2006) A rice Tc1/Mariner-like element transposes in yeast. Plant Cell 18:2469-2478

Yang G, Nagel DH, Feschotte C, Hancock CN, Wessler SR (2009) Tuned for transposition: molecular determinants underlying the hyperactivity of a Stowaway MITE. Sci 325:1391-1394

Yau YY, Santos K, Simon PW (2005) Molecular tagging and selection for sugar type in carrot roots using co-dominant, PCRbased markers. Mol Breeding 16:1-10 
Yu S, Li J, Luo L (2010) Complexity and specificity of precursor microRNAs driven by transposable elements in rice. Plant Biol Mol Rep 28:502-511
Zhou Y, Magill CW, Magill JM, Newton RJ (1998) An apparent of nonsymmetrical and sustained strand-specific hemimethylation in the $D c 8$ gene of carrot. Genome 41:23-33 